\title{
NUMERICAL ANALYSIS OF STRESS INTENSITY FACTOR AND T-STRESS IN PIPELINE OF STEEL P264GH SUBMITTED TO LOADING CONDITIONS
}

\author{
Hassane Moustabchir \\ Équipe Science et Ingénierie des Matériaux (ESIM), Département de Physique, Université My Ismaïl, Errachidia, Morocco; \\ e-mail: hmoustabchir74@gmail.com \\ JAmal Arbaoui \\ ENSTA Bretagne, LBMS/DFMS, Brest Cedex, France; e-mail: jamal.arbaoui@ensta-bretagne.fr
}

AzARi Zitouni

LaBPS, Université Paul Verlaine Metz,Ecole Nationale d'Ingénieurs de Metz, Metz, France

SAID HARIRI

TPCIM, Ecole des Mines de Douai, Douai Cedex, France

IHOR DMYTRAKH

Karpenko Physico-Mechanical Institute of National Academy of Sciences of Ukraine, Lviv, Ukraine

\begin{abstract}
Stress singularities occur at crack tips, corners and material interfaces. The stress intensity factors and T-stresses are coefficients of structural components where the active stress singular and first regular stress terms, respectively, are denoted by William's eigen function expansion series. A finite element analysis by CASTEM 2000 have been undertaken in order to determine the evolution of the T-stress and stress intensity factor terms in mode I for an arc of pipeline specimens with an external surface crack. A stress difference method described by Moustabchir et al. (2012) are adapted and, in the following step, the volumetric method is then embedded to compute the SIFs and T-stress near the crack tip. Different crack geometries combined with different length-to-thickness ratios are examined for the T-stress and stress-intensity factor. The revisited stress difference method employed here shows to be an accurate and robust scheme for evaluating the T-stress/SIFs in an arc of the pipeline.
\end{abstract}

Keywords: T-stress, Stress Intensity Factor (SIF), Finite Element Method (FEM), Stress Difference Method (SDM), volumetric method

\section{Introduction}

Fracture behavior of materials is generally characterized by a single parameter such as the SIF. Traditionally, the SIF has been used in the determination of initiation and propagation of cracks in brittle materials. However, the introduction of a second fracture parameter, as well as the T-stress, allows better understanding of the effect of structural and loading configuration at the crack tip, even that the physical significance of this parameter inserted is limited. To correlate the higher term effects with an appropriate physical parameter, a difficult task to simplify the higher terms to define the T-stress term was conducted (Nakamura and Parks, 1991; Du and Hancock, 1991). The T-stress is defined as constant stress acting parallel to the crack and its magnitude is proportional to the nominal stress in the vicinity of the crack. A positive T-stress strengthens the level of crack tip stress triaxiality and leads to high crack tip constraint; while a negative T-stress reduces the level of crack tip stress triaxiality and leads to the loss of the crack tip constraint. It was noted (Nakamura and Parks, 1991; Smith et al., 2001) that T-stress, which is the non-singular linear elastic stress component parallel to the crack, characterizes the 
local crack tip stress field for an elastic linear material, and the elastic plastic material with the restriction of small-scale yielding conditions.

In this paper, we revise the method described by Moustabchir et al. (2012) using directly single Finite Element (FE) analysis by CASTEM 2000 program. The Stress Difference Method (SDM) adapted here is developed to compute the elastic T-stress efficiently and accurately by evaluating $\left(\sigma_{x x}-\sigma_{y y}\right)$ at a point ahead of the crack tip. The powerful idea shows that the errors acquired in the numerical values of $\sigma_{x x}$ and $\sigma_{y y}$ near the of crack tip evolve with $x$, i.e. the distance from the crack tip, and their difference eliminates the errors effectively. For a homogeneous material, we calculate the T-stress using the difference of the normal stresses along $\theta=0$, i.e. $\left(\sigma_{x x}-\sigma_{y y}\right)$, which is a method that can lead to significant numerical errors due to the recovery of stresses very close to the crack tip. We then present a volumetric method for computing T-stress and stress intensity factor $K_{I}$ in mode I by modifying the stress difference method, the so-called Modified Stress Difference Method (MSDM). Details of the volumetric method were well explained by Pluvinage (2003). Condequently, in Section 4, we pay attention to it presenting a clear evidence that it agrees with our case studied. The physical meaning of the results is discussed, giving a better solution to the detection of the pipelines integrity.

\section{Finite element analysis}

In an isotropic linear elastic body containing a three-dimensional crack subject to a symmetric loading, the stresses developed in front of the crack for each dimension can be written as a series expansion (2012). Near the crack tip (see details of the crack tip in Fig. 1), the higher order terms of the series expansion are negligible, thus the stresses for mode I fracture can be written

$$
\begin{aligned}
\sigma_{x x} & =\frac{K_{I}}{\sqrt{\pi r}} \cos \frac{\theta}{2}\left(1-\sin \frac{\theta}{2} \sin \frac{3 \theta}{2}\right)+T & \sigma_{y y} & =\frac{K_{I}}{\sqrt{\pi r}} \cos \frac{\theta}{2}\left(1+\sin \frac{\theta}{2} \sin \frac{3 \theta}{2}\right) \\
\sigma_{z z} & =\frac{K_{I}}{\sqrt{\pi r}} 2 \nu \cos \frac{\theta}{2}+E \varepsilon_{z z}+\nu T & \sigma_{x y} & =\frac{K_{I}}{\sqrt{\pi r}} \sin \frac{\theta}{2} \cos \frac{\theta}{2} \cos \frac{3 \theta}{2} \\
\sigma_{x z} & =\sigma_{y z}=0 & &
\end{aligned}
$$

where $K_{I}$ is mode I local stress intensity factor, $E$ is Young's modulus and $\nu$ is Poisson's ratio. Here, $T$ is the elastic T-stress representing tension/compression acting parallel to the cracked plane.

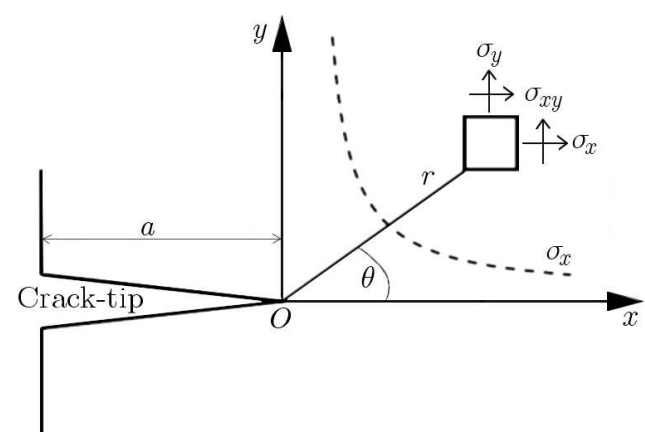

Fig. 1. Crack-tip cartesian coordinates $(x, y)$ and polar coordinates $(r, \theta)$

The subscripts $x, y$ and $z$ in Fig. 1 describe the local Cartesian co-ordinate system formed by the plane normal to the crack front and the plane tangential to the crack front point; $r$ and $\theta$ are the local polar co-ordinates. 
In mode I loading, equation (2.1) (state of $\sigma_{x x}$ ) shows that the stress comprises a singular term and the $T$ part

$$
T=\left(\sigma_{x x}-\sigma_{y y}\right)_{r=0, \theta=0}
$$

The T-stress developed varies with different crack geometries and loading. It plays the dominant role on shape and size of the plastic zone, while estimating the degree of local crack tip yielding, and also in quantifying the fracture toughness. In a particular case of $K_{I I}=0$, Mostafavi et al. (2010) stated that $T$ must be proportional to the reference stress $\sigma_{x x}$ and, therefore, it can be normalized to obtain biaxial stress ratios

$$
\beta=\frac{T \sqrt{\pi r}}{K_{I}}
$$

The finite element method has been used to determine the crack-tip parameters $T$ and $K$ for the an arc of pipe specimens. The structures are modeled by CASTEM 2000 code in two dimensions under plane strain conditions using free-meshed isoparametric quadrilateral elements, with quarter-point singularity elements at the crack-tip. Only one half of the test apparatus has been modelled due to symmetry in the geometry and loading conditions. The mesh generated for the elastic analyses comprises 31485 elements and 63526 nodes. The arc of pipeline fracture specimen geometry is illustrated in Fig. 2a. A fan-like mesh focused at the crack tip (on the plane perpendicular to the crack front, see Fig. $2 \mathrm{~b}$ ) is employed because this yields more accurate T-stress values. Furthermore, a detailed mesh sensitivity study has shown that further refinement

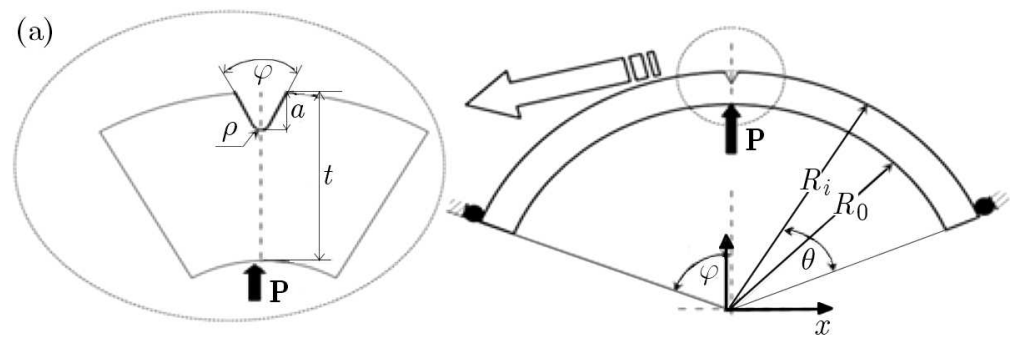

(b)

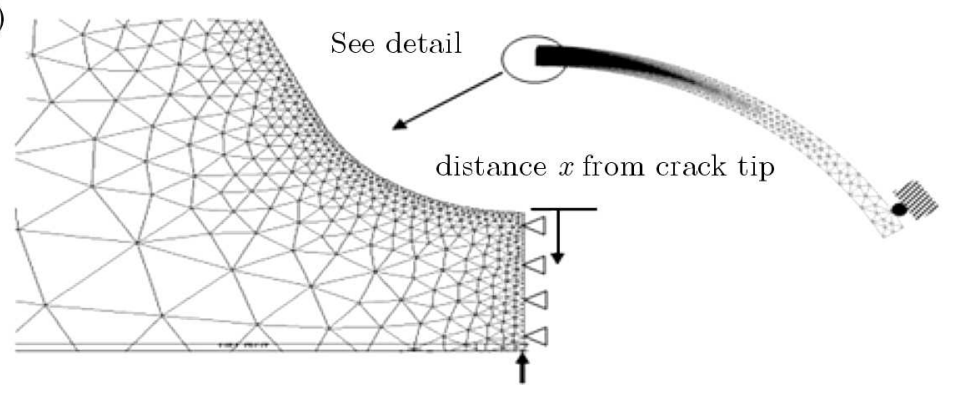

\begin{tabular}{|c|c|c|c|c|c|c|c|}
\hline$R_{i}[\mathrm{~mm}]$ & $P[\mathrm{~N}]$ & $\Phi\left[^{\circ}\right]$ & $\theta\left[^{\circ}\right]$ & $\varphi\left[{ }^{\circ}\right]$ & $\rho[\mathrm{mm}]$ & $a[\mathrm{~mm}]$ & $t[\mathrm{~mm}]$ \\
\hline \hline 219.55 & 150 & 60 & 60 & 45 & 0.15 & $1.22-4.88$ & 6.1 \\
\hline
\end{tabular}

Fig. 2. (a) Geometry of sample with boundary conditions and loading configuration using an arc of the pipe. (b) Typical 2D finite-element mesh used to model the cracked arc of the pipe for elastic analysis

of the mesh leads to small changes only $(<1 \%)$. The wall thickness of the pipeline is $6.1 \mathrm{~mm}$ and length $40 \mathrm{~mm}$. The specimen is loaded by a concentrate force $(P=150 \mathrm{~N})$ on the top of the TPB specimen in the symmetric plane. The support and the symmetric boundary conditions are also used in this model. The material used in this survey is steel P264GH. The stress/strain curve of the material is represented by the Ramberg-Osgood curve generated during the hardening processes and expressed as

$$
\frac{\varepsilon}{\varepsilon_{0}}=\frac{\sigma}{\sigma_{y}}+\alpha\left(\frac{\sigma}{\sigma_{y}}\right)^{n}
$$


In Eq. (2.4), $\varepsilon_{0}, \sigma_{y}, \alpha$ and $n$ are constants, and $E \varepsilon_{0}=\sigma_{y}$. A model that encloses deformation plasticity processes with only a small geometry change as well as continuum model is invoked. We take $\alpha=1$ and $\sigma_{y}=410 \mathrm{MPa}$. Two values of the strain hardening exponent $n$ are embedded, with the values of $n=1$ and $n=0.0446$. The case of $n=1$ corresponds to the elastic path. A series of finite element analyses are performed for different crack length ratios $a / t$. The chemical compositions of the material samples are included in Table 1, and the true stress versus strain curve that shows the behavior for this material are plotted in Fig. 3.

Table 1. Chemical composition of material specimens (weight \%)

\begin{tabular}{|l|c|c|c|c|c|c|c|}
\hline \multicolumn{1}{|c|}{ Material } & $\mathrm{C}$ & $\mathrm{Mn}$ & $\mathrm{S}$ & $\mathrm{Si}$ & $\mathrm{P}$ & $\mathrm{Al}$ & $\mathrm{Fe}$ \\
\hline \hline Tested steel & 0.135 & 0.665 & 0.002 & 0.195 & 0.013 & 0.027 & Bal. \\
\hline $\begin{array}{l}\text { Steel P264GH according to } \\
\text { Standard EN10028.2-92 }\end{array}$ & 0.18 & 1 & 0.015 & 0.4 & 0.025 & 0.02 & Bal. \\
\hline
\end{tabular}

Table 2. Mechanical properties of steel P264GH

\begin{tabular}{|l|c|}
\hline Young's modulus & $E=207000 \mathrm{MPa}$ \\
\hline Poisson's ratio & $\nu=0.3$ \\
\hline Yield stress & $\sigma_{y}=R_{e}=410 \mathrm{MPa}$ \\
\hline Ultimate tensile strength & $R_{m}=440 \mathrm{MPa}$ \\
\hline Elongation to fracture & $A=35 \%$ \\
\hline
\end{tabular}

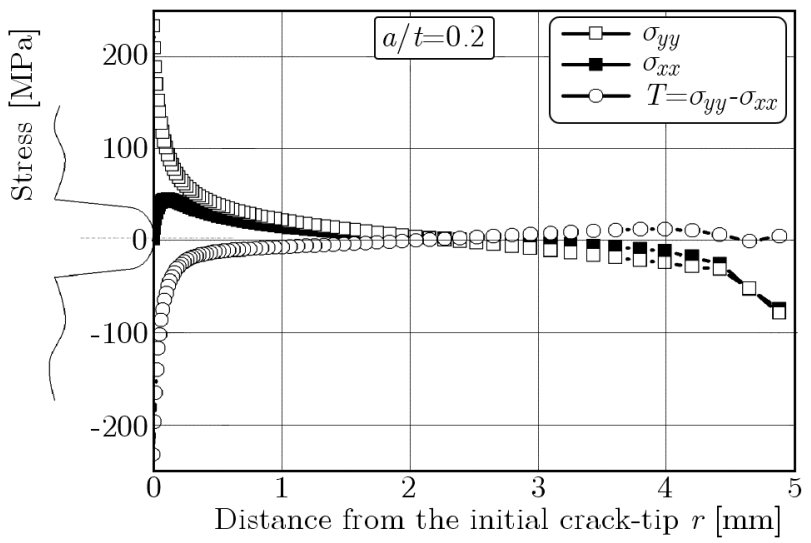

Fig. 3. T-stress definition by the stress difference method $(a / t=0.2)$

\section{Mode I stress intensity factors and T-stresses results}

The calculated mode I stress intensity factor $K_{I}$ and the T-stress are plotted in Fig. 4. T-stress variations presented in Fig. 3 (at the near crack tip $x<1 \mathrm{~mm}$ ) denote that $T$ is more sensitive for all cases and the results from the arc of pipe specimens show that the SDM does not provide a constant value of $T$. It first increases with the crack length and then decreases rapidly. Later, it decreases slowly along the distance $x$ for any ratio of $a / t$. When the ratio $a / t<0.4$ is reached and the distance from crack tip $x>0.23 \mathrm{~mm}$, the T-stress seems to remain constant at $0 \sim-25 \mathrm{MPa}$, while $T$ decreases strongly with the increase of the crack length when $a / t \geqslant 0.4$. The change of sign of the T-stress from positive to negative may be due to the fact that the magnitude of the local moment closing the crack increases with an increase in the crack depth. The negative values for the arc of pipe specimens indicate a low crack front constraint and an extended plastic 
deformation around the crack front. This conforms to the solutions of the T-stress for a 2D single edge crack under tension (Smith et al., 2001; Mostafavi et al., 2010; Moustabchir, 2012; Lu and Meshii, 2014). The T-stress becomes more negative when the free surface is closed due to the loss of crack-front constraint.

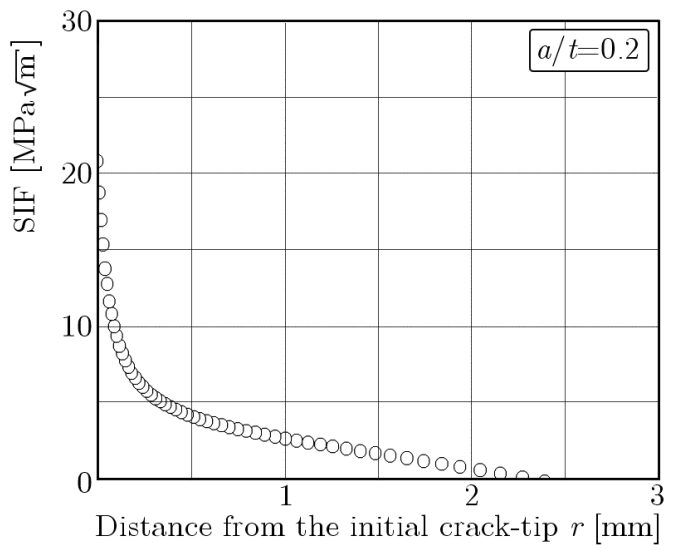

Fig. 4. An example of evaluation of different stresses for $a / t=0.2$

Notably, the T-stress has always the same value $(T=23.3 \mathrm{MPa})$ at a distance $x=0.24 \mathrm{~mm}$ for any given crack length. It is worth noting that the T-stress variations with the crack depth and the crack length for the bending loading case are in agreement with the results reported by Wang and Bell (2004) for plates and Jayadevan et al. (2005) (work devoted to studies on the pipes behavior). However, the variation of T-stress with crack depth in plates is just the reverse of that observed in pipes under bending (Wang, 2003). This is evidenced when the crack depthincrease, while the T-stress in pipes under bending decreases slowly and remains negative (see Fig. 3). It strongly increases for the plate from a negative to a high positive value (Wang, 2003). In Fig. 4, we show the results obtained from the bending loading. Variations of the SIF presented as the ratio of $d / t=36$ show that the decrease in the SIF with crack length is not monotonic as verified by $2 \mathrm{D}$ analyses. With an increasing of the ratio $a / t$, the SIF increases strongly at the crack tip (Fig. 4). The effect of crack length is more pronounced for short cracks compared to long cracks. Furthermore, for the shortest crack, an increase in the SIF with crack depth becomes marginal for the ratio $a / t=0.2$. The results were confirmed by the powerful research developed in works by Jayadevan et al. (2005).

Note that the stress intensity factors always increases for a distance of $x<0.115 \mathrm{~mm}$ for any ratios $a / t$ and then decreases along the crack length. $K$ always has a maximum at $x \approx 0.11$ for any given cracklength. In Fig. 4, we present the results obtained from variations of the SIF for deep cracks $(a / t=0.2-0.8)$. The SIF increases strongly with an increase in the crack length and becomes positive until reaching the maximum value for $a / t=0.6$, then decreases for negative values. Note that for $a / t=0.7$, the SIF vanishes.

In order to normalize the effect of the T-stress relatively to the stress intensity factor in mode I, Mostafavi et al. (2010) proposed in Eq. (2.3) a dimensionless parameter called the biaxiality ratio $\beta$. Figure 5 shows the variation of the biaxiality ratio versus the ratio of crack length-to-width $a / t$ for various distances near the tip-crack. The sign of the biaxiality ratio changes from positive to negative values as the distance from the crack tip increases. Near the crack tip and for the shortest crack, $a / t<0.4$, the ratio $T \sqrt{\pi a} / K_{I}$ decreases strongly along a certain distance and then reaches a positive value. On the other hand, for $a / t \geqslant 0.4$, T-stresses change from positive to negative values when $x<0.3 \mathrm{~mm}$. Considering a slight imperfection under mode I loading, Hadj Meliani et al. (2010) found that the crack path is stable for negative T-stresses and unstable for positive T-stresses. 


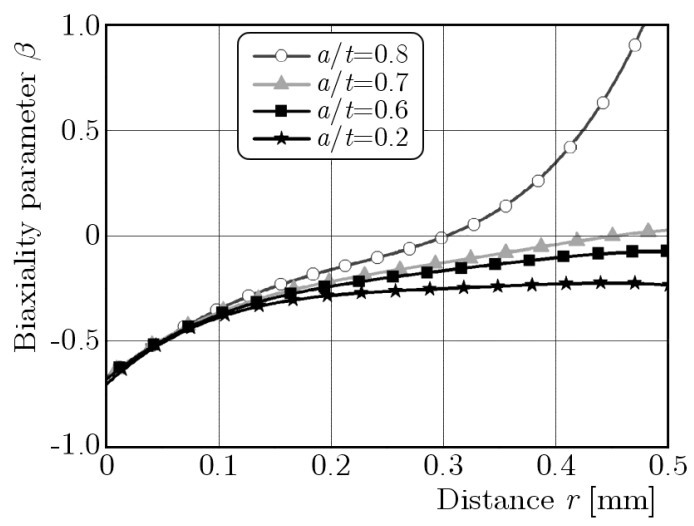

Fig. 5. Biaxiality ratio for an arc of the pipe specimen; (a) distribution of $T \sqrt{\pi a} / K_{I}$ along the crack length, (b) biaxiality ratio versus the crack length-to-width ratio $a / t$ for mode I near the tip crack

A distance from the crack tip greater than $0.3 \mathrm{~mm}$ is sufficient for most problems, except when $a / t$ is large, see Fig. 6. Fracture criteria that include the SIFs and T-stress can be implemented in the present code and used to predict the crack initiation angle. These remarks are in agreement with the results from well-known authors, see e.g. Ayatollah et al. (1998). The variations of the biaxiality ratio versus the crack length-to-width ratio $a / t$ for two specimens (SENB and SENT) published by Fett (1997) using the boundary collocation method, also the method proposed by Kim and Paulino (2003) using the FEM and by Sutradhar and Paulino (2004) employing the interaction integral method, are compared to the present results using an arc of a pipe subject to bending loading (Fig. 3). At the notch of the crack, Fig. 5 shows that the stress difference method (SDM) provides a constant value for $T \sqrt{\pi a} / K_{I}$ and the sign remains the same between 2.6 and 2.8 for any ratio $a / t$.

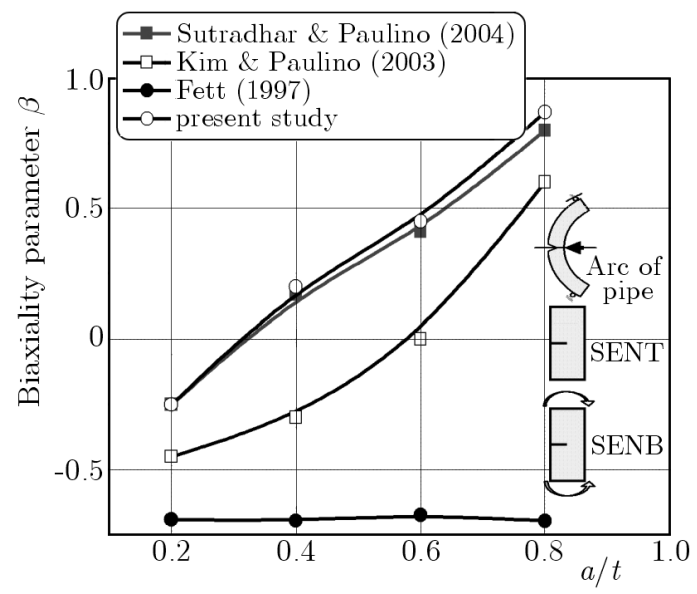

Fig. 6. Biaxiality ratio versus $a / t$ for various specimens at the notch of the crack

\section{Evaluation of the SIF and T-stress by the volumetric method}

The elastic and elastic-plastic normal stress intensity factor distribution at the notch tip exhibits a decreasing trend with distance from the notch tip. A careful analysis demonstrated for this configuration was initiated by Pluvinage (2003). It characterized three zones, as shown in Fig. 7: the first one is very near the notch tip where the normal SIF is practically constant and/or increasing to its maximum value $K_{I}^{\max }$, the intermediate zone and the third one are considered as the location where the pseudo stress intensity factor singularity can be simulated. 


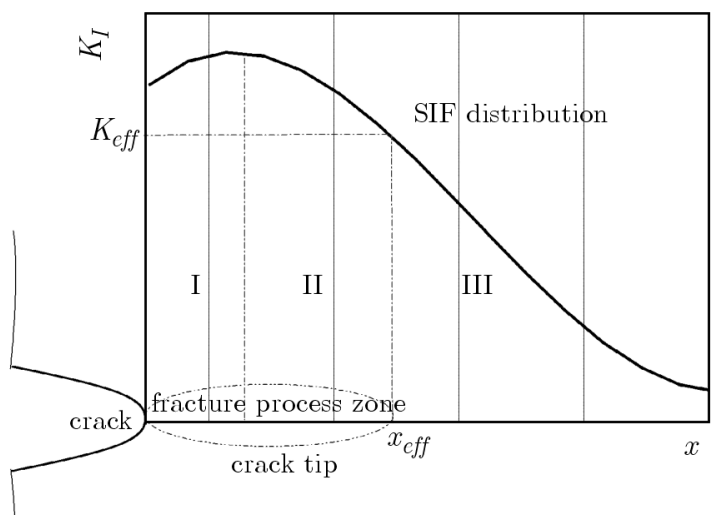

Fig. 7. Schematic SIF distribution at the notch tip for definition of fracture parameters - the effective SIF and effective distance

It is assumed that the fracture process needs a physical volume. This assumption is supported by the fact that fracture resistance is affected by the loading mode, structure geometry and scale effect. The values of the hot spot in the neighborhood of any point in the fracture process volume are taken into account. This volume is assumed to be quasi-cylindrical by analogy to the notch plastic zone, which has a similar shape. The diameter of this cylinder is called the effective distance $x_{\text {eff }}$. The effective stress intensity factor and the effective T-stress can be estimated by averaging the value of SIF distribution along this effective distance (Pluvinage, 2003).

Although many works have estimated the stress intensity factors with the presence of T-stress of an arc of a pipe, they have exclusively used the fracture mechanics to estimate the toughness. According to recent investigations, other methods are applied to predict the T-stress and stress intensity factors. One of these methods, named the volumetric approach, is concerned with the modification of the Stress Difference Method (SDM). The volumetric approach is a macromechanical method and it uses an elastic-plastic distribution and the stress intensity factor gradient evolution to predict the stress intensity factor. The main idea of this method is the application of the effective stress intensity factor to the region near notch roots and examination of intrinsic characteristics of this small size as well as the essential zone by consideration of numerical SIF results including non-linear behavior of materials.

\section{Concluding remarks and extensions}

- In mode I, the T-stress can be obtained by direct use of the stress difference method (SDM) along the crack. The SDM, applied to evaluate the T-stress and SIFs, provides an accurate and robust scheme for calculating the fracture parameters.

- The numerical results obtained by CASTEM 2000 are in good agreement with known results for single cracks. In general, the T-stress computations are more time-consuming than those for SIFs. This observation is in agreement with analogous studies in the FEM fields.

- The results for $T$ are strongly highlighted near the crack tip and far away from the crack tip, and depend on the geometrical parameters.

- In general, the T-stress has larger domain dependence or contour dependence than the stress intensity factors or the J-integral. The data for T-stresses as well as mode I stress intensity factor $K_{I}$ are represented by simple fit relations.

- The ratio obtained from the numerical results by several researchers showed some difference from the predicted stress intensity factor in mode I with and without presence of the T-stress. It can be reasonably predicted from the regression result and using the relation- 
ship $\left(K_{I} / K_{I C}\right)^{2}+\alpha\left(T / T_{\text {crit }}\right)^{2}$ where $\alpha$ is an empirical constant and $T_{\text {crit }}$ is the critical T-stress.

- The data for T-stresses as well as for mode I stress intensity factor are represented by simple fit relations.

\section{References}

1. Ayatollah M.R., Pavier M.J., Smith D.J., 1998, Determination of T-stress from finite element analysis for mode I and mixed mode I/II loading, International Journal of Fracture, 91, 283-298

2. CASTEM 2000.http://www-cast3m.cea.fr/cast3m/index.jsp

3. Du Z.-Z., HANCOCK J.W., 1991, The effect of non-singular stresses on crack-tip constraint, Journal of the Mechanics and Physics of Solids, 39, 555-567

4. Fetт T., 1997, A Green's function for T-stresses in an edge cracked rectangular plate, Engineering Fracture Mechanics, 57, 365-373

5. Hadj Meliani M., Azari Z., Pluvinage G., Matvienko Yu.G., 2010, The effective T-stress estimation and crack paths emanating from U-notches, Engineering Fracture Mechanics, 77, 1682-1692

6. Jayadevan K.R., Thaulow C., Østby E., Berg B., Skallerud B., Holthe K., Nyhus B., 2005, Structural integrity of pipelines: T-stress by line-spring, Fatigue and Fracture of Engineering Materials and Structures, 28, 467-488

7. Kim J.-H., Paulino G.H., 2003, T-stress, mixed-mode stress intensity factors, and crack initiation angles in functionally graded materials: a unified approach using the interaction integral method, Computer Methods in Applied Mechanics and Engineering, 192, 1463-1494

8. Lu K., Meshin T., 2014, Three-dimensional T-stresses for three-point-bend specimens with large thickness variation, Engineering Fracture Mechanics, 116, 197-203

9. Mostafavi M., Smith D.J., Pavier M.J., 2010, Reduction of measured toughness due to outof-plane constraint in ductile fracture of aluminium alloy specimens, Fatigue and Fracture of Engineering Materials and Structures, 33, 724-739

10. Moustabchir H., Azari Z., Hariri S., Dmytrakh I., 2012, Three-dimensional t-stress to predict the directional stability of crack propagation in a pipeline with external surface crack, Key Engineering Materials, 498, 31- 41

11. Nakamura T., Parks D.M., 1991, Determination of elastic T -stress along three-dimensional crack fronts using an interaction integral, International Journal of Solids and Structures, 29, 1597-1611

12. Pluvinage G., 2003, Fracture and Fatigue Emanating from Stress Concentrators, Kluwer Publisher, Springer link. 978, 1-4020

13. Smith D.J., Ayatollahi M.R., Pavier M.J., 2001, The role of T-stress in brittle fracture for linear elastic materials under mixed-mode loading, Fatigue and Fracture of Engineering Materials and Structures, 24, 137-150

14. Sutradhar A., Paulino G.H., 2004, The simple boundary element method for transient heat conduction in functionally graded materials, Computer Methods in Applied Mechanics and Engineering, 193, 4511-4539

15. WANG X., 2003, Elastic T-stress solutions for semi-elliptical surface cracks in infinite thickness plates, Engineering Fracture Mechanics, 70, 731-756

16. WAng X., Bell R., 2004, Elastic T-stress solutions for semi-elliptical surface cracks infinite thickness plates subject to non-uniform stress distributions, Engineering Fracture Mechanics, 71, $1477-1496$ 